\title{
Chryseobacterium indologenes
}

National Cancer Institute

\section{Source}

National Cancer Institute. Chryseobacterium indologenes. NCI Thesaurus. Code C86260.

A species of aerobic, Gram negative, rod shaped bacteria in the phylum Bacteroidetes.

This bacteria is nonmotile, catalase and oxidase positive, non-fermentative, indole positive, hydrolyzes starch and can grow on cetrimide agar. C. indologenes is most commonly found in soil and water and is a rare pathogen, associated with meningitis in neonates and premature infants and nosocomial infections in adults. 